langages, information, médiations

Parcours de recherche en SIC: littérature scientifique, méthodes et terrain

\title{
Quelles approches possibles du terrain chinois en communication organisationnelle? Situation et retour d'expérience
}

Possible Approaches to the Chinese Field of Organizational Communication?

Situation and Feedback

Mylène Hardy

\section{OpenEdition}

Journals

Édition électronique

URL : http://journals.openedition.org/edc/946

DOI : $10.4000 /$ edc. 946

ISSN : 2101-0366

Éditeur

Université Lille-3

Édition imprimée

Date de publication : 1 avril 2009

Pagination : 109-128

ISBN : 978-2-917562-01-7

ISSN : 1270-6841

\section{Référence électronique}

Mylène Hardy, «Quelles approches possibles du terrain chinois en communication organisationnelle? Situation et retour d'expérience », Études de communication [En ligne], 32 | 2009, mis en ligne le 01 avril 2011, consulté le 01 mai 2019. URL : http://journals.openedition.org/edc/946 ; DOI : 10.4000/edc.946

Ce document a été généré automatiquement le 1 mai 2019.

(C) Tous droits réservés 


\title{
Quelles approches possibles $\mathrm{du}$ terrain chinois en communication organisationnelle? Situation et retour d'expérience
}

\author{
Possible Approaches to the Chinese Field of Organizational Communication? \\ Situation and Feedback
}

Mylène Hardy

1 La Chine attire de plus en plus de chercheurs en sciences sociales, qu'ils souhaitent comprendre son évolution socio-économique ou accompagner des organisations étrangères s'y implantant. Le terrain chinois est un terrain difficile, de par les différences culturelles et linguistiques tout d'abord, mais également par la relation spécifique entre société et Etat, à laquelle doivent s'adapter les chercheurs.

2 Le but de cet article est d'effectuer un état des lieux de la recherche en communication organisationnelle en Chine, et d'examiner les différentes méthodes de recherche envisageables, à partir de la littérature et de notre expérience (observation des pratiques de recherche, examen de la littérature chinoise et entretiens informels avec des professeurs et étudiants chinois). La revue de l'organisation académique des chercheurs en communication organisationnelle en Chine permet de souligner l'aspect dispersé de la recherche et le manque de recherches sur la communication organisationnelle. Par ailleurs, l'on note un recours abondant aux méthodes quantitatives et la faiblesse de l'utilisation des méthodes qualitatives parmi les méthodes de recherche généralement utilisées pour étudier la communication organisationnelle en Chine. Le retour sur notre expérience de deux années passées en Chine permet d'examiner les difficultés que nous avons rencontrées en les reliant aux spécificités du pays, mais aussi à la situation générale des jeunes chercheurs sur le terrain. 


\section{Aperçu de la recherche en communication organisationnelle en Chine}

\section{La naissance de la discipline « communication » en Chine}

3 Les sciences de l'information et de la communication, sont en Chine un ensemble de disciplines constitué sous une forme apparentée à celle que l'on trouve aux États-Unis, mais avec quelques variations :

- une discipline de sciences de l'information apparentée à l'informatique voire aux télécommunications, et comme en France ou aux États-Unis à tout ce qui concerne la gestion de l'information (archivage, documentation, etc.) ;

- une discipline de sciences de la communication, mais nommée en chinois 传播学, chuanboxue, c'est-à-dire «sciences de la transmission », et qui en fait regroupe des chercheurs en communication de masse.

4 Le terme de chuanbo, transmission, a été importé en Chine depuis les États-Unis au début des années 80, à l'ouverture du pays. Il a alors concurrencé le terme de xinwenxue, journalisme, qui couvrait alors les recherches en communication, ce qui a conduit à la création d'un nouveau champ, plus large: celui de xinwen chuanboxue Comme le remarque Sun (2002, pp. 8-9), «It is significant to realize that although researchers know the etymological relation of 'community' to 'communication' as well as the social implications of 'communication' in Western culture, few of them pay enough attention to the semantic and cultural differences between chuanbo and 'communication'. They examine the Chinese history and practice of communication in light of 'chuanbo' instead of 'communication'. This reflects the traditional orientation and emphasis of Chinese communication, that is, the flow of information from a center to margins rather than the sharing of information within a community ". Il faut dire que l'histoire de la communication en Chine a été mouvementée, à en croire les auteurs qui en retracent le parcours (Liao, 2003 ; Sun, 2002). Après avoir été introduite à l'université Fudan en 1982, elle s'est développée comme une discipline d'enseignement, car c'était là les objectifs principaux proposés dans la première convention nationale sur les communications. De son introduction en Chine jusqu'au milieu des années 80 , en effet, la discipline s'est construite autour de l'importation et de l'évaluation de ce qui se faisait à l'étranger, alors qu'ensuite les chercheurs se concentrés sur son contenu et son développement mêmes (Liu, 1993). À partir de 1986, avec la seconde convention, les objectifs ont été de développer la théorie, à partir des concepts occidentaux, mais en tentant de les adapter à la Chine, et de se centrer sur l'étude des médias et de la communication de masse. Cependant, les chercheurs se heurtèrent aux factions les plus à gauche du parti communiste chinois qui défendaient l'idée d'un contrôle du Parti sur les médias. Lors de Tiananmen, les enseignements en communication furent accusés d'avoir joué un rôle dans le soulèvement et la discipline fut classée comme «bourgeoise ", ce qui mena à sa quasi-disparition. Ce n'est qu'en 1992 que la communication fut réhabilitée et que les conventions purent être poursuivies. À partir de 1993, le département de communication de l'Académie des Sciences Sociales de Chine étendit à tout le territoire la convention annuelle de communication. En 1997, la cinquième convention se pencha sur les critères et les méthodes de recherche en communication. Finalement, ce n'est qu'en 1998 que le gouvernement autorisa les universités à délivrer des grades de Master et de Doctorat en communication et que la communication s'installa officiellement comme 
discipline. Une histoire d'une vingtaine d'années contre presque cent pour les Etats-Unis explique les différences de structuration disciplinaire. Dans ce cadre, l'on comprend aussi mieux le manque de publications diversifiées dans la discipline. Par ailleurs, la situation est tout simplement semblable à celle qu'ont connue les SIC en France ou dans d'autres pays: en réalité, les études en communication interpersonnelle ne sont en général pas effectuées par des chercheurs en communication, mais par d'autres disciplines, et souvent par des chercheurs étrangers :

- la psychosociologie, discipline apparue récemment en Chine, et qui s'intéresse aux problèmes relationnels et à la gestion de la «face » (Zeng \& Liu, 2004 ; Shao, 1988);

- la linguistique et le rôle de la communication dans les relations interculturelles (Dou, 2005 ; Gao, 1998 ; Freeman \& Habermann, 1996);

- la sociologie et la communication comme éléments des relations sociales dans le capital social (exemple : Zhou, 2005).

Powers (2002, p. 37) fait donc le constat d'une discipline disparate: "Research on the communication practices of Chinese people has been burgeoning in recent years. However, despite the large body of literature, constructing a comprehensive understanding of Chinese communication phenomena is a daunting task, partially because the literature is scattered across many disciplines and partially because no conceptual map exists of how the studies might contribute to a more coherent understanding of Chinese communication». Cependant, ces dernières années, la discipline s'organise et se constitue de plus en plus autour des mêmes axes qu'aux Etats-Unis : communication de masse, toujours majoritaire, mais aussi communication interpersonnelle, communication interculturelle et communication des organisations.

\section{Les recherches actuelles en communication organisationnelle chinoise}

6 La communication organisationnelle est l'axe le moins abouti de la discipline: la recherche chinoise dans ce domaine est rare du fait de son existence encore très jeune (Chen \& Lin, 2003). Comprise dans le même sens qu'aux États-Unis la plupart du temps, c'est-à-dire comme communication s'effectuant à l'intérieur d'une organisation, en général une entreprise, elle est étudiée de manière assez dispersée selon les axes suivants :

- la communication marketing (très étudiée) (Chan \& Cheng, 2002);

- les relations publiques et la communication externe de l'entreprise (Chen, 2007) ;

- la négociation (Kong, 1995).

7 La communication interne de l'entreprise est peu étudiée par les chercheurs chinois en tant que telle. En général, leurs ouvrages ou articles ne font que reprendre les résultats et thèses développés par les chercheurs américains, recèlent rarement des résultats d'enquêtes, et sont parfois plutôt destinés à un public de praticiens (He, Hou \& Ren, 2002). Dans tous les cas, la communication organisationnelle est étudiée par :

- des chercheurs en science de gestion, qui souvent utilisent la communication comme variable unique, définie par des critères de vitesse de transmission (Sun \& Li, 2005) ;

- des chercheurs en linguistique et/ou spécialistes d'un pays, qui s'interrogent sur la communication organisationnelle dans un cadre comparatif ou interculturel. C'est le cas par exemple des recherches que l'équipe du professeur Z. Lihua a mené dans le Guangdong sur 
les entreprises à capitaux mixtes, et qui sont connues en France par la collaboration qui a été effectuée avec D. Desjeux (Zheng \& Desjeux, 2002).

D'autres recherches dispersées où apparait la communication ont été conduites par des sociologues ou des anthropologues. Une étude intéressante est par exemple celle de la situation interculturelle de A. Hanser (2007), qui décrit notamment, dans son observation participante dans deux grands magasins chinois, sa communication avec ses collègues chinoises, sa hiérarchie et les clients du magasin. Dans ces études cependant, la communication ne constitue qu'une petite partie du propos.

9 Le sujet commence à présent à attirer l'attention des chercheurs, notamment grâce aux efforts d'un universitaire qui tente d'en structurer les connaissances et la recherche : $\mathrm{H}$. Hening, professeur à l'Université de technologie de Chine, dans le département des communications et politiques technologiques. Avec ses étudiants, il semble tenter de poser les bases de la mise en place d'une étude de la communication organisationnelle en Chine, puisque ses articles sont soit des revues de littérature américaine sur la communication organisationnelle $(\mathrm{Hu} \& \mathrm{Hu}, 2008 ; \mathrm{Hu}, 2007)$, soit des réflexions sur la méthodologie (Hu, 2007), la définition (Hu \& Ye, 2004b), ou encore une réflexion sur l'histoire de la recherche en communication organisationnelle en Chine (Hu \& Hu, 2008; $\mathrm{Hu} \& \mathrm{Ye}, 2004 \mathrm{a})$.

Selon $\mathrm{Hu}$, "la période de 2005 à aujourd'hui constitue le tout début du développement de la communication organisationnelle chinoise » ( $\mathrm{Hu} \& \mathrm{Hu}, 2008$, p.36). Le tableau 1, proposé par $\mathrm{Hu}$ et $\mathrm{Hu}$ (2008), montre bien la différence entre les études portant sur la communication de masse et les autres champs d'étude en communication, et la progression de la communication organisationnelle ces dernières années.

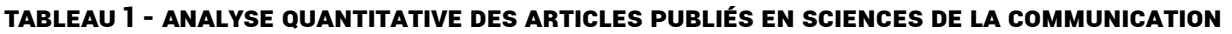
(TRADUIT DE HU \& HU, 2008, P.36)

\begin{tabular}{|l|l|l|l|l|l|l|l|l|l|l|}
\hline & $\begin{array}{l}\text { Avt } \\
2000\end{array}$ & 2000 & 2001 & 2002 & 2003 & 2004 & 2005 & 2006 & 2007 & Total \\
\hline $\begin{array}{l}\text { Communication } \\
\text { interpersonnelle }\end{array}$ & 24 & 5 & 5 & 4 & 10 & 10 & 5 & 9 & 30 & 124 \\
\hline Communication de groupe & 1 & 1 & 0 & 0 & 0 & 0 & 0 & 0 & 3 & 7 \\
\hline $\begin{array}{l}\text { Communication } \\
\text { organisationnelle }\end{array}$ & 5 & 1 & 4 & 0 & 2 & 2 & 6 & 7 & 14 & 53 \\
\hline Communication de masse & 283 & 42 & 37 & 32 & 53 & 52 & 52 & 54 & 82 & 717 \\
\hline
\end{tabular}

11 Le tableau permet de voir d'ailleurs que l'étude de la communication interpersonnelle a commencé à se développer au même moment que la communication organisationnelle, et que les études sur la communication de groupe se développent tout juste. Cependant, une analyse quantitative simple comme celle proposée ici ne donne pas un aperçu suffisant de la littérature. En effet, de prime abord, l'on pourrait penser que 53 articles publiés donneraient un bon aperçu de la recherche en communication organisationnelle en Chine. Le problème est cependant que la plupart des articles font soit une revue de la littérature américaine en notant justement le manque de recherches en Chine $(\mathrm{Hu} \& \mathrm{Hu}$, 
2008 ; Huang, 2001 ; Pan, 1994), soit n'effectuent que des développements théoriques, sans données empiriques, quantitatives ou qualitatives (Tong, 2007 ; Hu, 2006). Ce point vaut aussi pour la communication interpersonnelle chinoise, car comme le notent Gao et Xiao (2002, pp.22-23) : «Data-based studies of human communication are still difficult to find ».

\section{Les méthodes de recherche en communication organisationnelle}

12 Les facteurs soulevés ci-dessus influencent l'orientation de la recherche et donc les méthodes préconisées. Si méthodes de recherche quantitatives sont connues et utilisées, la recherche chinoise a moins approfondi les méthodes qualitatives. C'est donc ce domaine, dans lequel l'on peut espérer un plus grand développement à l'avenir, sur lequel nous avons choisi de nous attarder plus particulièrement ici. Avant d'examiner les différents types de méthodes, nous rappelons quelques éléments communs à toute méthode et qui méritent attention pour toute recherche en Chine :

- l'influence culturelle sur la question de recherche posée. Cette question, qui mérite d'être évoquée étant donné l'eurocentrisme de nombreuses recherches en communication sur la Chine (Chuang \& Hale, 2002), ne sera pas abordée ici, notre communication portant sur des problèmes plus proprement méthodologiques ;

- le problème de l'échantillonnage ;

- le développement d'instruments de recherche adaptés ;

- le défi de la collecte des données ;

- le problème de l'interprétation des données (Stening \& Zhang, 2007).

Certains de ces problèmes ressortent plus dans certains types de recherche que dans d'autres.

\section{Méthodes quantitatives expérimentale et empirique}

Issues d'une tradition positiviste américaine et considérant encore la communication sous l'angle du code et des effets du message, les recherches en communication en Chine sont quasiment exclusivement des recherches quantitatives, expérimentales (notamment en marketing) ou empiriques (le plus souvent par questionnaire). La volonté d'améliorer la qualité scientifique de la recherche a en effet reposé sur l'introduction des méthodes quantitatives à la fin des années 90 (Liao, 2003). Comme en sociologie, le problème qui se pose pour les questionnaires est de savoir quelle est la représentativité de l'informant. Pour la communication organisationnelle, le problème se pose de manière encore plus prégnante, du fait de la nouveauté de la rencontre entre le monde de l'entreprise et celui de la recherche. Selon les expériences rapportées par les chercheurs chinois eux-mêmes (Huang, 2007), les questionnaires peuvent poser problème car ils sont souvent remplis par la secrétaire de la personne visée. Il faudrait peut-être alors, comme le préconisent certains chercheurs, administrer le questionnaire sous forme d'entretiens dirigés. En général en tout cas, le problème des méthodes empiriques par questionnaires réside dans la collecte des données, surtout lorsqu'il s'agit d'étudier un grand échantillon d'entreprises. 


\section{L'enquête par entretien}

15 Les entretiens sont donc une autre forme assez courante d'enquête en sociologie comme en sciences de gestion en Chine. L'avantage des entretiens est de pouvoir maîtriser qui est l'informant. Cependant, cette relation directe avec l'informant fait disparaître la possibilité d'anonymat existant dans les questionnaires, anonymat qui peut faciliter certaines réponses et même la formulation de certaines questions. En Chine, ce point pèse particulièrement sur la recherche. Dans cette culture où l'on ne donne pas d'information aux inconnus de "l'out-group ", l'entretien peut être perçu avec plus de méfiance que le questionnaire. Par conséquent, certaines questions seront éludées. Si la question des jeux de face de l'informant et celle de ne pouvoir poser certaines questions directement se posent dans toute recherche par entretien, elles sont beaucoup plus accentuées en Chine où les habitudes culturelles de communication sont déterminées là encore par l'appartenance ou non à l'«in-group ", c'est-à-dire au cercle restreint de personnes proches de l'informant (en général, famille et amis) (Gao, 2006). Avec les membres de l'« in-group », la communication est directe et le « je » peut laisser entrevoir ses pensées. Avec les membres de l'«out-group ", la communication est de type polie, utilisant notamment le détour comment moyen d'expression (Gao, 2006; Gao, Ting-Toomey \& Gudykunst, 1996). La qualité des entretiens risque donc paradoxalement dépendre du degré d'intimité entre le chercheur et les informants, ou en tout cas, entre le chercheur et l'intermédiaire qui a mis en relation le chercheur et les informants. Si les informants font confiance à l'intermédiaire, une partie de cette confiance sera aussi attribuée au chercheur, ce qui lui facilitera la tâche. La confiance s'installant dans le long terme, la meilleure solution consisterait donc à passer plusieurs années sur un terrain, en ne commençant réellement les entretiens qu'au bout d'un ou deux ans. Cela dépend également du type d'entretien effectué. Les récits de vie sont ainsi souvent plus faciles à étudier et certaines recherches sur les entreprises chinoises sont effectuées à partir des récits de vie des entrepreneurs (Guilheux, 2007).

\section{Les méthodes ethnographiques}

S'il existe des enquêtes par interview, il existe peu, à notre connaissance d'enquêtes de terrain de type ethnographique impliquant de l'observation (Lee, 2007), et encore moins utilisant des enregistrements de conversation en milieu naturel, même public, en Chine. La communication et la sociologie s'intéressent peu à l'entreprise. En sciences de gestion, les démarches de type ethnographique sont quasi-inexistantes. Cela est dû tout autant à la non-reconnaissance des données qualitatives comme base de recherche qu'à la difficulté d'entrer sur le terrain. La non-reconnaissance des recherches qualitatives est due à l'histoire de la recherche scientifique du pays. Les chercheurs chinois ont longtemps effectué des recherches de type descriptif. Ces recherches, où la subjectivité était assumée, ne s'interrogeaient pas sur les méthodes de recherche ni sur les critères de scientificité ou d'objectivation. La volonté du pays d'être reconnu sur le plan scientifique a créé un élan positiviste en sciences sociales, où les méthodes quantitatives reprises des traditions américaines ont été posées comme les seules méthodes assurant une scientificité. S'est imposé et s'impose alors encore aujourd'hui une dichotomie entre quantitatif, critère de scientificité, et qualitatif, symbole de médiocrité scientifique. Hormis en sociologie où une réflexion sur la manière de rendre scientifiques des 
recherches qualitatives s'est posée, notamment à l'école de sociologie de l'université Tsinghua, dont certains professeurs reprennent les méthodes françaises de rechercheaction (Merle, 2004), peu de réflexions approfondies ont été menées dans d'autres disciplines comme en sciences de gestion sur l'utilité des recherches qualitatives et sur la manière de les mettre en œuvre sur le terrain proprement chinois, ainsi que nous l'ont rapporté plusieurs professeurs chinois rencontrés sur le terrain. Cependant, l'intérêt de plus en plus grand porté en Chine à l'anthropologie (comme discipline) (Croll, 2006) permet de penser qu'une plus grande diffusion de ses méthodes en communication et en gestion aura lieu dans les prochaines années en Chine. La démarche anthropologique des sinologues français (par exemple les recherches anthropologiques de T. Pairault en économie, et de J.-L. Rocca ou I. Thireau en sociologie) devrait y aider.

\section{Les méthodes de recherche-action}

Paradoxalement, les méthodes de recherche-action, dont une partie sont qualitatives, sont depuis longtemps ancrées comme méthodes de recherche en Chine. S'appuyant sur une longue tradition praxéologique (Bloom, 1979) et réactualisée par Mao (qui a écrit un essai sur le rapport entre connaissance et pratique, 1937), la recherche-action a été développée par les chercheurs chinois avec l'aide d'organisations internationales dans le domaine de l'éducation rurale ou de l'administration publique (Hughes \&Yuan, 2005), ou en sociologie pour aider les victimes des expropriations dans le cadre des plans d'urbanisation des grandes villes chinoises (Shen, 2005). Concernant les entreprises, nous avons trouvé très peu d'études impliquant de la recherche-action (Wang, 1989). La recherche-action semble pourtant bien convenir à des recherches en communication organisationnelle en Chine. Les entreprises chinoises souffrent d'un manque de communication et de coopération entre les employés (Hardy-Zhang \& Agostinelli, 2008; Chen, 2005). La recherche-action, qui pourrait permettre une meilleure prise de conscience des acteurs, rendrait en même temps possible une observation approfondie des pratiques, voire d'autres formes de recherches en communication organisationnelle. Bien que la situation évolue, notre expérience nous a cependant montré la limite de l'acceptation des chercheurs étrangers par les entreprises chinoises (Kane, 1995 ; Lindsay et Dempsey, 1983), du moins lorsque la recherche est menée en solitaire. Nous proposons donc de relater cette expérience comme exemple des obstacles qui peuvent intervenir et limiter la recherche de terrain en Chine.

\section{Retour d'expérience}

\section{Première immersion dans la recherche en Chine}

Doctorante en Sciences de l'information et de la communication, nous n'avons décidé de commencer notre thèse qu'après deux ans passés à travailler en Chine, qui nous avaient permis d'entrevoir notre sujet et d'ores et déjà la difficulté de le mettre en œuvre s'il était appréhendé par des méthodes ethnographiques. Portant sur l'intelligence économique, donc sur la relation entre information et stratégie de l'entreprise, il touchait en effet à deux domaines sensibles pour toute entreprise, de quelque pays qu'elle soit. Juste avant de repartir en Chine, nous avions rencontré un jeune sociologue français en sociologie du travail qui en revenait, et qui s'était vu reprocher à sa soutenance de thèse de n'avoir pas 
réussi à pénétrer l'entreprise qui lui servait de terrain et d'avoir dû se contenter d'effectuer des entretiens avec les employés à l'extérieur de l'entreprise. Il nous avait dit : « Tu vas voir, c'est impossible d'entrer pour nous étrangers dans les entreprises, là-bas. Les recherches sur le milieu du travail sont trop sensibles ». Nous souhaitions tout de même relever le défi, nous donnant deux ans pour parvenir à trouver au moins une entreprise à observer. Si les entreprises chinoises ont beaucoup été étudiées par questionnaires, notamment au niveau micro-économique, il existe très peu de recherches ethnographiques sur elles, ni en France, ni en Chine, et nous pensions que si nous arrivions à relever le défi, notre contribution scientifique serait plus intéressante. Malheureusement, notre université n'avait pas de coopération avec des équipes de chercheurs chinois qui puissent nous aider dans notre démarche, ce qui constitua un premier obstacle sur notre route. Postulant à la bourse de recherche franco-chinoise, nous indiquâmes sur le formulaire le nom d'une université chinoise partenaire de la nôtre dans laquelle nous connaissions déjà un professeur, mais le gouvernement chinois nous envoya dans une autre université, inconnue de la nôtre, et l'on nous assigna également un directeur de recherche en sciences de gestion, que l'on estimait être plus proche de notre sujet de recherche. Malheureusement pour nous, ce directeur de recherche ne pouvait nous aider. Outre le fait que son axe de recherche était très différent du nôtre, il ne connaissait pas du tout les recherches de type qualitatif et ne proposait à ses étudiants que des recherches empiriques par questionnaires soumis à des étudiants en MBA. Le fait de n'avoir jamais été recommandée par notre directeur de recherche chinois à d'autres professeurs qui nous auraient à leur tour recommandée a été un grand handicap. Nos essais pour nous débrouiller seule n'étaient pas concluants, malgré l'absence d'obstacle linguistique majeur. Ainsi, nous avions demandé durant la pause d'un colloque à un de nos autres professeurs s'il pouvait nous présenter des professeurs susceptibles de nous donner des conseils pour notre recherche, mais le professeur nous avait regardée étonné, sans répondre. En Chine, en effet, d'après ce que nous ont appris nos observations et nos discussions avec nos camarades chinois, un doctorant ne peut réellement discuter avec des professeurs d'université, ni même aux heures de pause : aux repas des colloques, par exemple, les professeurs mangent à part et la hiérarchie est strictement conservée. Le seul moyen pour un doctorant français de pouvoir discuter avec des professeurs chinois est que son directeur de recherche français ait des relations avec des professeurs chinois, car ainsi la relation s'effectue sur le même plan hiérarchique. Un doctorant canadien, en sciences politiques, nous avait expliqué que son directeur de recherche canadien était un ami d'un professeur de l'université chinoise où nous nous trouvions, si bien que ce dernier l'avait accueilli à bras ouverts et lui avait déjà fait rencontrer d'autres professeurs dans d'autres universités ainsi que des hommes politiques spécialistes de son sujet.

\section{La difficulté d'entrer sur le terrain}

19 Dans notre cas, le seul moyen de trouver un terrain était soit d'effectuer de l'observation participante sous couvert de travailler ou d'essayer de passer par des relations pour nous faire recommander auprès d'une entreprise. L'observation participante est une solution souvent utilisée par les chercheurs enquêtant dans le domaine du travail en Chine (Hanser, 2007; Zheng, 2007). Nous envisagions la première solution en dernier recours seulement, car nous aurions difficilement trouvé un travail qui nous permette d'effectuer des observations sur notre sujet de recherche, l'intelligence économique, et que, si nous avions effectué ce choix, nous n'aurions pas pu effectuer notre enquête de manière 
ouverte, à cause du caractère sensible du sujet. Comme nous souhaitions poursuivre une carrière scientifique en Chine, nous ne voulions pas utiliser des méthodes déontologiquement problématiques, et ceci d'autant plus que notre sujet de recherche était sensible. Nous tentâmes de mettre en œuvre la deuxième solution en nous appuyant sur nos relations personnelles, mais les maigres résultats que nous obtînmes ne correspondaient pas à nos critères de recherche en termes de taille de l'organisation. Ce fut finalement le professeur que nous connaissions qui nous permit de trouver et de négocier un terrain, bien que la taille de l'échantillon reste également problématique pour la validité interne de la recherche. Nous dûmes également revoir nos objectifs : à la différence du jeune sociologue que nous avions rencontré avant de partir en Chine, nous pouvions effectuer les entretiens à l'intérieur de l'entreprise. Malheureusement, c'était la seule chose qui nous était accordée, bien que le professeur qui nous ait recommandée ait de proches relations avec le directeur. Pas d'observation possible en dehors de celles effectuées furtivement lors des interviews. Une des deux entreprises que nous avons pu observer ne nous a permis que d'aller dans la salle de réunion, quelqu'un nous y accompagnant toujours, de telle sorte que nous ne pouvions aller voir ce qui se passait en dehors; pourtant, dans cette entreprise, nous avions une très bonne relation avec le directeur qui par un heureux hasard travaillait parallèlement dans l'université où nous étudions et nous considérait donc comme un «membre de la famille». Ne pouvant pas enregistrer de communications verbales en situation réelle, nous décidâmes de créer une simulation et d'enregistrer les participants pour avoir un échantillon minimal de conversations. Nous créâmes également un certain nombre de méthodes graphiques dans nos entretiens pour interroger les personnes sur les situations de communication concernant notre sujet dans l'entreprise. Ainsi, partie de méthodes d'enquête très classiques, nous dûmes adapter nos méthodes d'investigation au fur et à mesure des obstacles qui se posaient sur notre chemin.

\section{L'influence de la nationalité sur la recherche}

La nationalité a par ailleurs beaucoup joué dans les difficultés que nous avons rencontrées dans notre recherche, à la fois parce que nous devions tenter d'éviter l'ethnocentrisme dans nos observations, et parce que le rapport communicationnel entre Chinois/ chercheurs étrangers est différent du rapport Chinois/chercheur chinois : bien que nous parlâmes chinois, le fait d'être étrangère nous faisait catégoriser comme appartenant au cercle le plus éloigné de "l'out-group ", celui des étrangers, envers qui les normes culturelles inculquent de montrer un visage lisse, sans 'information', c'est-à-dire envers qui la communication sera effectuée sous la forme la plus implicite et la plus indirecte. Bien qu'il soit beaucoup plus facile à présent que par le passé pour des chercheurs étrangers d'entrer sur des terrains chinois grâce à la réforme politique actuelle, comme nous le faisaient remarquer certains employés en me disant que "maintenant on a le droit de vous parler ", la négociation du terrain, surtout s'il est sensible, est difficile. Le comportement du professeur chinois qui nous a aidée est en cela révélateur. Au début, lorsqu'il nous présentait aux chefs d'entreprise pour essayer de "vendre" notre recherche, il expliquait que nous aimions beaucoup la Chine; puis, quand nous nous sommes mariée avec un Chinois, il nous a présentée en disant que nous aimions tant la Chine que notre mari était chinois, ce qui a facilité les démarches. Enfin, il a proposé à une de ses étudiantes chinoises de nous accompagner dans nos interviews pour faciliter les entretiens. En réalité, la présence de cette étudiante était indispensable. Certains des 
interviewés ne nous regardaient jamais pendant les entretiens, préférant répondre aux questions que nous posions en s'adressant uniquement à l'étudiante chinoise. L'un d'entre eux était si hostile que nous avons décidé de former l'étudiante chinoise à notre guide d'entretien pour qu'elle puisse nous aider à poser les questions à cette personne, elle devenant l'intervieweuse, et nous prenant des notes. Dans toute recherche sur un terrain étranger, dans tous les cas, il est intéressant de collaborer avec des chercheurs locaux, afin de permettre la triangulation des observations (Tsui, 2004). Nous interrogions ainsi régulièrement cette étudiante chinoise sur ses observations durant nos interviews, $y$ compris les observations sur notre propre attitude dans l'interaction. Nous avons aussi chargé cette étudiante, après l'avoir formée, d'une partie du travail d'animation lors de la séance de simulation, afin de mettre les participants en confiance. Cette partie de la recherche est d'ailleurs ce qui a le mieux fonctionné.

\section{Conclusion. Des difficultés inhérentes à la Chine?}

21 L'on peut légitimement se demander si les difficultés de mise en œuvre de la recherche sur le terrain chinois sont particulières à la Chine, comme l'ont décrit d'autres jeunes chercheurs avant nous (Seather, 2003 ; Stone, 1997). Elles ne le sont, selon nous, qu'en partie seulement.

\section{L'entrée sur le terrain}

Le problème de l'entrée sur le terrain des entreprises n'est pas du tout spécifique au pays. Un professeur chinois qui profite de ses missions de consultant pour effectuer des observations nous a expliqué que seule la renommée du professeur lui permettra d'être appelé par les entreprises qui, demandeuses, sont plus facilement prêtes à se laisser observer (Huang, 2007). En réalité, cela est vrai dans tous les pays: la renommée de certains laboratoires en sociologie des organisations ou en gestion permettant ainsi aux membres du laboratoire de trouver plus facilement un terrain. La difficulté est accrue en Chine par le fait le monde de l'entreprise commence tout juste à se développer et à ouvrir ses portes à l'extérieur. Il n'est pas habitué à recevoir des chercheurs, et encore moins des chercheurs travaillant sur la communication.

\section{Les relations avec les chercheurs locaux}

La recherche est un système socio-économique aux caractéristiques diverses selon les pays. A la différence de ce qui se fait en Occident, les relations entre doctorants et professeurs est très hiérarchisée en Chine, ce qui ne permet pas l'insertion facile d'un doctorant, a fortiori étranger, dans le système de recherche. Deux facteurs nous semblent importants dans ce cadre : le temps et le statut. Au bout de trois ans de relations avec nos enseignants et camarades chinois, nous sommes accueillie parmi eux comme dans une "famille ». Le fait qu'à présent, nous soyons docteur et occupions un poste à leur yeux important nous y aide beaucoup. Cela ne résout cependant pas entièrement les difficultés, car bonne part de nos difficulté viennent d'un excès de confiance dans notre capacité à entrer sur le terrain. 


\section{Le choix d'un sujet faisable}

En effet, l'ambition qui était la nôtre de contribuer à la recherche par la nouveauté des méthodes utilisées se doublait d'un espoir de pouvoir travailler sur un sujet nouveau et sensible dans le pays (l'intelligence économique) et sur un objet de recherche (l'entreprise) lui aussi relativement nouveau. Cet excès de volonté d'originalité ne pouvait pas mener à une recherche sans obstacles. Ces difficultés nous ont amenée à revoir notre théorie de départ, en nous obligeant à recadrer une théorie trop ambitieuse sur les parties observables.

Le terrain chinois est un terrain quasiment vierge en communication organisationnelle, et, étant donné que la littérature chinoise comporte très peu de données de terrain, toute contribution dans ce domaine est déjà intéressante. Il est donc intéressant pour le chercheur, avant de choisir un sujet, d'examiner non seulement ses propres objectifs, mais aussi le système socio-économique du terrain étranger dans lequel il va s'insérer, les besoins de ce système, et ses limites. D'où la nécessité, pour les appréhender et les dépasser, de la bonne connaissance de la recherche déjà effectuée par les chercheurs locaux, et d'un dialogue scientifique approfondi avec eux.

\section{BIBLIOGRAPHIE}

Bloom, A.H., (1979), The Impact of Chinese Linguistic Structure on Cognitive Style, Current Anthropologlogy, 20 (3), pp. 585-586.

Chan, K. \& Cheng, H., (2002), One Country, Two Systems: Cultural Values Reflected in Chinese and Hong-Kong Television Commercials, Gazette, The International Journal for Communication Studies , 64 (4), pp. 385-400.

Chen, L., (2005), Qiye neibu goutong zhong xinxi chuandi wenti yanjiu [Research on Information Transmission Issue of Communication within the Enterprises], Doctorate Degree Thesis in Management Science and Engineering, China: Central South University.

Chen, L. \& Lin, X., (2003), Guanli goutong lilun fazhan jieduan lueshu [On history-developing stages of the management communication], Zhongnan Daxue Xuebao (shehui kexue ban) [Journal of Central South University (Social Science)], 9 (6), pp. 812-1815.

Chen, X.H., (2007), Gonggong guanxixue xueyuan de chuanboxue fenxi [A Mass-communicative Analysis of the Disciplinary Nature of the Public Relation Research], Hubei daxue xuebao (Zhexue shehui kexue ban) [Journal of Hubei University (Philosophy and Social Science Edition)], 34 (3), pp. 120-124.

Chuang, R.L. \& Hale, C., (2003), A Critical Examination of the Eurocentric Representation of Chinese Communication, in Jia, Wenshan (dir.), Chinese Communication Theory and Research : Reflections, New Frontiers and New Directions, Westport, Greenwood, pp. 67-84.

Croll, E.J., (2006), New Research Trends in Anthropology/Sociology in China, China Aktuell, 3, pp. 90-97. 
Cui, J.Y., (2006), Zuzhi de guanli goutong yanjiu [Managerial Communication in Organization], Doctorate Degree Thesis in Labor Economics, China: Capital University of Economics and Business.

Dou, W.L., (2005), Kuawenhua Shangwu jiaoji /Intercultural Business Communication. Beijing, Gaodeng jiaoyu chubanshe [High Education Press].

Freeman, N.H. \& Habermann, G.M., (1996), Linguistic Socialization : A Chinese Perspective, Chap. 6, in M.H. Bond (dir), The Handbook of Chinese Psychology, Hong-Kong, Oxford University Press, pp. 79-92.

Gao, G., (2006), Core Concepts and Organizing Principles of Chinese Communication and Identity, China Media Research, 2 (2).

Gao, G., (1998), «Don't take my word for it ». Understanding Chinese speaking practices, International Journal of Intercultural Relations, 22 (2), pp. 163-186.

Gao, G., Ting-Toomey, S. \& Gudykunst, W.B., (1996), Chinese Communication Processes, Chap. 18 in M.H. Bond (dir), The Handbook of Chinese Psychology, Hong-Kong, Oxford University Press, pp. 282-293.

Guilheux, G., (2007), The Promotion of a New Calculating Chinese Subject : the case of laid-off workers turning into entrepreneurs, Journal of Contemporary China, 16 (50), pp. 149-171.

Hansen, C., (1985), Chinese Language, Chinese Philosophy, and «Truth», The Journal of Asian Studies, 44 (3), pp. 491-519.

Hanser, A., (2007), A Tale of Two Sales Floors, Changing Service-Work Regimes in China in C.K. Lee (2007), Working in China, Ethnographies of Labor and Workplace Transformation, London, Routledge, pp. 77-97.

Hardy-Zhang, M. \& Agostinelli, S., (2008), La dimension culturelle des pratiques communicationnelles de veille stratégique et son influence sur les systèmes de veille : l'exemple des entreprises chinoises, SIIE Conference 2008, 14-16/02/2008, in A. Flory, M. Ghenima et S. Sidhom (dirs.), Système d'information et intelligence économique, Tunis, IHE, pp. 249-262.

He, Y.Y., Hou, L. \& Ren, H.B, (2002), Xinxi goutong zhong jingliren yinggai zunhun de « 531 » celue [The « 531 » Tactics that Managers Should Follow in Their Working Communications], Zhongguo nongye daxue xuebao (Shehui kexue ban) [Journal of China Agricultural University (Social Sciences Edition)], 4, pp. 54-58.

Hu, Y.Y., \& Hu. H.N., (2008), Woguo zuzhi chuanbo yanjiu de fazhan licheng : 20 nian lai zuzhi chuanbo yanjiu zongshu (1988-2007) [Le développement de la recherche en communication organisationnelle en Chine : Revue de la recherche de ces 20 dernières années (1988-2007)], Jin Chuanmei [Today Media], 06, pp. 36-37.

Hu, H.N., (2007), Zuzhi chuanboxue yanjiu de fangfa yu shijiao [Méthodes et approches de la communication organisationnelle], Xinwen yu chuanbo yanjiu [Journalism et Communication Research], 01, pp. 62-66.

Hu, H.N., (2006), Zuzhi chuanbo fuhao de lunli yiyun [Le sens moral des symboles en communication organisationnelle], Shehui yanjiu zhanjian [Pratique de la recherche sociale], 4, pp. 192-194

Hu, H.N., \& Ye, Y.Z., (2004a), Zuzhi chuanboxue de fazhan zhuangkuang yu tixi goutian [Développement et construction du domaine de la communication organisationnelle], Xueshujie [Academic World], 05, pp. 262-269. 
Hu, H.N., \& Ye, Y.Z., (2004b), Zuzhi chuanboxue de jieding jiqi yiyi [Définition et sens de la communication organisationnelle], Zhongguo renmin daxue xuebao [Journal of People University], 06, pp. 130-136

Huang, W.W., (2007), Qiye ketexu chengzhang de jiben celue (yi Huawei wei anli) [Politique de base du développement durable des entreprises (Huawei comme exemple)], Conférence donnée le 29/05/2007 à la Faculté des Sciences Economiques et de Gestion de l'Université Tsinghua, Pékin, Chine.

Huang, X.J., (2001), Zuzhi chuanbo de yanjiu moshi ji sikao [Théorie et modèle d'étude pour la communication organisationnelle], Zhejiang Daxue Xuebao [Journal of Zhejiang University (Humanities and Social Sciences)], 11 (5), pp. 112-117.

Hughes, I. \& Yuan, L., (2005), The Status of Action Research in the People's Republic of China, Action Research, 3 (4), pp. 383-402.

Jia, W.S., (dir.) (2002), Chinese Communication Theory and Research : Reflections, New Frontiers and New Directions, Westport, Greenwood, 281 p.

Kane, K., (1995), An Inside View of Management in a People's Republic of China Global Enterprise, Journal of Management Inquiry, Déc., pp. 381-387.

Kong, Z.L., (1995), Tanpan yu siwei fangshi [Négociation et mode de pensée], Beijing, Jingji kexue Chubanshe [Editions des Sciences économiques], $186 \mathrm{p}$.

Lindsay, C.P. \& Dempsey, B.L., (1983), Ten Painfully Learned Lessons About Working in China: The Insights of Two American Behavioral Scientists, The Journal of Applied Behavioral Science, 19 (3), pp. 265-276.

Liao, S.Q., (2003), 20 shiji 90 niandai de zhongguo dalu chuanboxue yanjiu. [The study in communication in Mainland China in the 1990'], Fudan Xuebao (shehui kexue ban) [Fudan Journal (Social Sciences)], 1, pp. 124-129.

Liu, M.N., (1993), Chuanboxue xuyao chuanbo : di san ci quanguo chuanboxue yantaohui ceji [Il faut communiquer sur la communication; une vue de biais sur la $3^{\text {ème }}$ conférence nationale de communication], Xinwen Zhishi [Savoir journalistique], 8, pp. 31-33.

Lu, X., (dir.) (2002), Chinese Communication Studies : Contexts and Comparisons, Westport, Greenwood, $250 \mathrm{p}$.

Mao, Z.D., (1937), On practice: On the relation between knowledge and practice, between knowing and doing, http://www.marxists.org/reference/archive/mao/selected-works/volume-1/ mswv1_16.htm, date de la dernière visite : 20/06/2007.

Merle, A., (2004), Vers une sociologie chinoise de la « civilisation communiste ». Perspectives chinoises, 81, janvier-février, pp. 4-15

Pan, Y.P., (1994), Jianli zhongguo tese de zuzhi chuanboxue [Construire une théorie de la communication organisationnelle aux caractéristiques chinoises], Xinwen Daxue [Journalistic University], 2, pp. 12-15.

Saether, E., (2003), Fieldwork as a learning process. Abstract from Workshop on Fieldwork Methodology and Practice in China, Copenhagen, 2-3 October 2003, Nordic Institute of Asian Studies (NIAS), http://www.nias.ku.dk/chinafieldwork/abstracts.htm, date de la dernière visite: 10/11/2007.

Shen, Y., (2005), Intervention sociologique en Chine : deux cas d'études. Conférence donnée à l'Antenne expérimentale franco-chinoise des Sciences Sociales, 10/11/2005, Pékin, Chine.

Stening, B. W. \& Zhang, M. Y., (2007), Methodological Challenges Confronted when Conducting Management Research in China, International Journal of Cross-Cultural Management, 7 (1), pp. 121-142. 
Stone, E., (1997), From the Research Notes of a Foreign Devil: Disability Research in China, in Barnes, C. et Mercer, G. Doing Disability Research, Leeds, The Disability Press, http://www.leeds.ac.uk/ disability-studies/books/book3.htm, date de la dernière visite: 15/10/2007.

Sun, Y.F. \& Li, Y., (2005), Jiyu zuzhi neibu goutong yu zenghe nengli de neibu zhishi zenghe yu chuangxin [Intraorganizational Communication and Integration Capability and Knowledge Combination], Zhongguo guanli kexue [Chinese Journal of Management Science], 13, Special Issue, Oct., pp. 56-61.

Tong, C., (2007), Lun zuzhi chuanboxue yu guanlixue de lianxi [Sur le rapport entre communication organisationnelle et sciences de gestion]. Anhui Wenxue [Anhui Literature], 12, pp. 229-230.

Tsui, A.S., (2004), Contributing to Global Management Knowledge : The Case for High Quality Indigeneous Research, Asia Pacific Journal of Management, 21, pp. 491-513.

Wang, H.M., (1989), Zhongguo qiye zhongde xingdong yanjiu he zuzhi fazhan celue. [Action Research and O.D. Strategies in Chinese Enterprises], Yingyong Xinlixue [Chinese Journal of Applied Psychology], 1.

Wu, Y.M., (2003), Jiangnan jiqichang chanquan zhidu ji zuzhi jiegou de yanjiu [Etude des droits de propriété et de la structure organisationnelle de la manufacture de machines de Jiangnan], Master Degree Thesis in Management, China, Shanghai Maritime University.

Zheng, T.T., (2007), From Peasant Women to Bar Hostesses: An Ethnography of China's Karaoke Sex Industry, in C.K. Lee (2007), Working in China, Ethnographies of Labor and Workplace Transformation, London, Routledge, pp. 124-144.

Zheng, L.H. \& Desjeux, D., (dirs.) (2002), Entreprise et vie quotidienne en Chine. Approches Interculturelles. Paris, L'Harmattan.

Zhou, J.G., (2005), Zhongguoren renji guanxi de jiegou yu gongneng fengxi [Analyse de la structure et capacité des relations interpersonnelles des Chinois], Shanghai, Shanghai Sanlian Shudian [Shanghai Sanlian Bookstore].

\section{RÉSUMÉS}

Cet article examine les différentes méthodes de recherche envisageables en communication organisationnelle en Chine, à partir de la littérature et d'un retour d'expérience, en trois parties. Dans la première, nous proposons un court aperçu de l'organisation académique des chercheurs en communication en Chine, en mettant en avant l'aspect dispersé de la recherche et le manque de recherches sur la communication en entreprise. Nous examinons dans un deuxième temps les méthodes de recherche généralement utilisées pour étudier la communication organisationnelle en Chine et soulignons les besoins en méthodes qualitatives. Nous résumons dans une dernière partie notre expérience en Chine, en mettant en avant les difficultés que nous avons rencontrées sur le terrain.

Using the literature and her own experience, the author examines the existing research methods in organizational communication and their applicability to the Chinese fieldwork. First, the Chinese academic organization in the field of communication is examined and the lack of research on organizational communication is outlined. Then the author reviews existing methods in organizational communication in China and shows the lack of qualitative methods. The last part describes the author's own experience on the Chinese fieldwork and highlights the problems she has encountered there. 
INDEX

Mots-clés : communication organisationnelle, Chine, méthodologie, terrain

Keywords : organizational communication, China, methodology, fieldwork

\section{AUTEUR}

MYLĖNE HARDY

Mylène Hardy est docteure en Sciences de l'Information et de la Communication. (laboratoire GERIICO) Elle est actuellement attachée de coopération pour la coopération linguistique et éducative auprès de l'Ambassade de France en Chine, à Pékin. Ses recherches portent sur la communication organisationnelle en Chine, notamment sur le rapport entre information et stratégie dans les entreprises chinoises. 